\title{
TU/e emonownen

\section{Finite-time learning control using frequency response data with application to a nanopositioning stage}

\section{Citation for published version (APA):}

de Rozario, R., Fleming, A., \& Oomen, T. (2019). Finite-time learning control using frequency response data with application to a nanopositioning stage. IEEE/ASME Transactions on Mechatronics, 24(5), 2085-2096. [8777112]. https://doi.org/10.1109/TMECH.2019.2931407

\section{Document license:}

TAVERNE

DOI:

10.1109/TMECH.2019.2931407

Document status and date:

Published: 01/10/2019

\section{Document Version:}

Publisher's PDF, also known as Version of Record (includes final page, issue and volume numbers)

\section{Please check the document version of this publication:}

- A submitted manuscript is the version of the article upon submission and before peer-review. There can be important differences between the submitted version and the official published version of record. People interested in the research are advised to contact the author for the final version of the publication, or visit the $\mathrm{DOI}$ to the publisher's website.

- The final author version and the galley proof are versions of the publication after peer review.

- The final published version features the final layout of the paper including the volume, issue and page numbers.

Link to publication

\section{General rights}

Copyright and moral rights for the publications made accessible in the public portal are retained by the authors and/or other copyright owners and it is a condition of accessing publications that users recognise and abide by the legal requirements associated with these rights.

- Users may download and print one copy of any publication from the public portal for the purpose of private study or research.

- You may not further distribute the material or use it for any profit-making activity or commercial gain

- You may freely distribute the URL identifying the publication in the public portal.

If the publication is distributed under the terms of Article 25fa of the Dutch Copyright Act, indicated by the "Taverne" license above, please follow below link for the End User Agreement:

www.tue.nl/taverne

Take down policy

If you believe that this document breaches copyright please contact us at:

openaccess@tue.nl

providing details and we will investigate your claim. 


\title{
Finite-Time Learning Control Using Frequency Response Data With Application to a Nanopositioning Stage
}

\author{
Robin de Rozario ${ }^{(0)}$, Andrew Fleming ${ }^{(}$, and Tom Oomen ${ }^{(1)}$
}

\begin{abstract}
Learning control enables significant performance improvement for systems that perform repeating tasks. Achieving high tracking performance by utilizing past error data typically requires noncausal learning that is based on a parametric model of the process. Such model-based approaches impose significant requirements on modeling and filter design. The aim of this paper is to reduce these requirements by developing a learning control framework that enables performance improvement through noncausal learning without relying on a parametric model. This is achieved by explicitly using the discrete Fourier transform to enable learning by using a nonparametric frequency response function model of the process. The effectiveness of the developed method is illustrated by application to a nanopositioning stage.
\end{abstract}

Index Terms-Iterative learning control, frequency response, motion control, nanopositioning.

\section{INTRODUCTION}

$\mathbf{L}$ EARNING from error data that are obtained from past operations is an effective approach to improve the performance of mechatronic systems that perform repetitive tasks, including nanopositioning devices [1], industrial printers [2], and additive manufacturing systems [3]. More specifically, repetitive control (RC) can be applied to reduce tracking error in applications that operate in a continuous fashion [4], [5], whereas iterative learning control (ILC) enables performance improvement for systems that operate in a batch-to-batch manner [6]-[9].

Parametric models are often employed in the design of RC and ILC controllers to achieve high convergence speeds. Fast

Manuscript received July 30, 2018; revised March 18, 2019; accepted July 10, 2019. Date of publication July 26, 2019; date of current version October 15, 2019. Recommended by Technical Editor Q. Zou. This work was supported in part by Océ Technologies and in part by the Netherlands Organization for Scientific Research through Research Program VIDI under Project 15698. (Corresponding author: Robin de Rozario.)

R. de Rozario and T. Oomen are with the Faculty of Mechanical Engineering, Control Systems Technology, Eindhoven University of Technology, 5600 MB Eindhoven, The Netherlands (e-mail: r.d.rozario @tue.nl; t.a.e.oomen@tue.nl).

A. Fleming is with the Department of Electrical and Computer Engineering, University of Newcastle, Callaghan, NSW 2308, Australia (e-mail: andrew.fleming@newcastle.edu.au).

Color versions of one or more of the figures in this paper are available online at http://ieeexplore.ieee.org.

Digital Object Identifier 10.1109/TMECH.2019.2931407 convergence is obtained when the learning filter closely approximates the inverse system dynamics [10], [11]. Consequently, typical learning filter design approaches are based on inverting a parametric plant model [12]. However, special measures are required to ensure that the inverse of a nonminimum phase (NMP) model is stable. More specifically, NMP zeros imply that a causal and stable inverse of the system does not exist, and that a stable inverse is instead anticausal [13]. For an anticausal inverse, the input corresponding to a desired output can be determined through a process known as stable inversion [14], though determining the exact inverse response requires infinite previewing of the desired output. Consequently, methods that approximate inverse linear time-invariant (LTI) dynamics with a finite amount preview have received considerable attention [12], [15]-[17]. Since these methods are inherently model based, significant requirements are imposed on learning filter design in terms of parametric identification and approximate inversion.

Iterative inversion-based control (IIC) is a related method that avoids the need for parametric models and seems to overcome the limitations related to bounded inversion. Aside from the robustness filter that was initially omitted in IIC [18], the IIC method [19] appears theoretically similar to infinite-time frequency-domain ILC [20], yet the two methods differ substantially in their implementation. In ILC, updating of the input signal is typically performed by using filtering operations, whereas in IIC, the updating is performed by using the discrete Fourier transform (DFT). The latter enables the use of nonparametric frequency response function (FRF) models, thereby removing the need for parametric models. This approach is shown to be effective [21]-[23] and seems to inherently facilitate noncausal learning with bounded inputs [24], [25], yet a full analysis of the potential limitations imposed by NMP zero dynamics has not yet been established.

In practice, the IIC method is typically implemented in discrete finite time and is reported to accommodate both batchto-batch and continuous processes. Pre-existing analyses of IIC assume that the system is in a steady state. This can be approximately achieved in batch-to-batch processes by including trailing zeros [19] or by measuring only the final periods of a periodic task [21]. In this way, updating the input can be performed offline, as is characteristic of ILC. Similarly, measuring the steady state of a continuous process can be approximately achieved by waiting for some time after the input 
was last updated [26]. In this way, the input can be updated, while the process operates continuously, as is characteristic of $\mathrm{RC}$. In practice, however, the process is never truly in the steady state, and pre-existing analyses do not provide clear guidelines to address the waiting period that is required to avoid the instability incurred by residual transients.

Although significant progress has been made to reduce the modeling and design requirements in learning control, at present, a systematic approach for fast and robust learning for NMP systems is not yet established. The aim of this paper is to fill this gap by developing a finite-time FRF-based learning control framework, supported by key analysis that are lacking in the present literature. This is achieved in combination with the following contributions.

C1) A method is developed to stably invert an NMP LTI system by using its FRF (see Section III).

C2) A robust finite-time IIC (FT-IIC) approach is developed (see Section II), and guidelines are derived to achieve high performance and robustness against uncertainties (see Section IV).

C3) A finite-time analysis of the developed approach is established to determine the waiting period that is required to achieve convergence, and it is shown how the developed approach relates to ILC and RC (see Section V).

C4) The effectiveness of the developed method is confirmed by applying it to control a nanopositioner in the continuous implementation setting (see Section VI).

These contributions extend existing IIC as follows. First, contribution $\mathrm{C} 1$ motivates the use of FRF models in learning by showing that stable inversion of NMP systems can readily be achieved in the frequency domain [14], [27]. Second, contribution $\mathrm{C} 2$ incorporates robustness by explicitly considering convergence in the presence of uncertainties in the FRF data. Third, contribution C3 provides guidelines for the effective implementation of IIC in finite time by considering the effect of the transient response on the convergence.

This paper extends the initial research reported in [18] by significantly extending contribution $\mathrm{C} 2$, adding contributions $\mathrm{C} 1$ and $\mathrm{C} 3$, and by providing rigorous proofs of the results.

\section{A. Notation}

The sets of real numbers, complex numbers, and integers are denoted by $\mathbb{R}, \mathbb{C}$, and $\mathbb{Z}$, respectively. The nonnegative real line, the unit circle, and the complex right half plane are denoted by $\mathbb{R}_{>0}=[0, \infty), \mathbb{C}^{\circ} \triangleq\{\xi \in \mathbb{C}|| \xi \mid=1\}$ and $\mathbb{C}_{>0} \triangleq\{\xi \in$ $\mathbb{C} \mid \Re\{\xi\}>0\}$. For a discrete time (DT) signal $f: \mathbb{Z} \mapsto \mathbb{R}$, the norms of $f$ are defined as $\|f\|_{p}^{p}=\sum_{t=-\infty}^{\infty}|f(t)|^{p}$ and $\|f\|_{\infty}=$ $\max _{t \in \mathbb{Z}}|f(t)|$, and $f$ is called $N$-periodic if $f(t+N)=f(t)$ $\forall t \in \mathbb{Z}$, with $N \in \mathbb{N}$. The spaces with bounded $\|\cdot\|_{p}$ and $\|\cdot\|_{\infty}$ norms are denoted by $\ell_{p}$ and $\ell_{\infty}$. The discrete time Fourier transform (DTFT) and its inverse are denoted by $\mathcal{F}_{\infty}$ and $\mathcal{F}_{\infty}^{-1}[28$, Sec. 2.12]. The DFT and its inverse are denoted by $\mathcal{F}_{N}$ and $\mathcal{F}_{N}^{-1}$ and are defined as $S(k)=\mathcal{F}_{N}\{s(t)\} \triangleq \frac{1}{\sqrt{N}} \sum_{t=0}^{N-1} s(t) e^{-j \omega_{k} t}$, $s(t)=\mathcal{F}_{N}^{-1}\{S(k)\} \triangleq \frac{1}{\sqrt{N}} \sum_{k=0}^{N-1} S(k) e^{j \omega_{k} t}, \quad \omega_{k}=\frac{2 \pi k}{N}, \quad k=$ $0,1, \ldots, N-1$ [28, Sec. 2.21]. Moreover, $[A]_{i j}$ denotes the

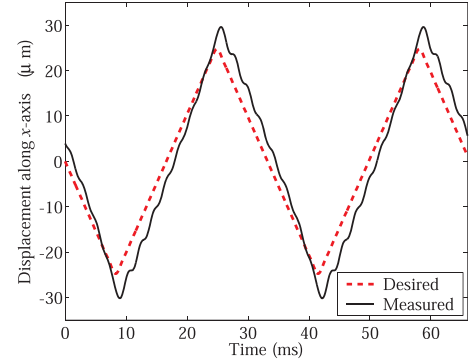

(a)

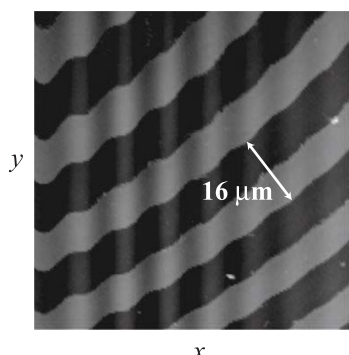

(b)
Fig. 1. Effect of positioning errors on the image quality in AFM. (a) Actual and desired probe displacement over time. (b) Distorted image (the actual features are parallel lines) [30, Sec. 2.6].

$i j$ th element of the matrix $A \in \mathbb{C}^{n \times m}, A^{H} \triangleq \bar{A}^{T}$, with $\bar{A}$ being the complex conjugate of $A$, and $\bar{\rho}(A)$ and $\bar{\sigma}(A)$ represent the largest eigenvalue and largest singular value of $A$. Additionally, $\operatorname{diag}\{s(k)\}$ is a diagonal matrix whose $k$ th diagonal equals $s(k)$. In this paper, a single-input single-output LTI DT system $G$ is considered, which is represented by a minimal realization

$$
G:\left[\begin{array}{l|l}
A & B \\
\hline C & D
\end{array}\right]= \begin{cases}x(t+1) & =A x(t)+B u(t) \\
y(t) & =C x(t)+D u(t)\end{cases}
$$

where $x(t) \in \mathbb{R}^{n_{x}}, u(t), y(t) \in \mathbb{R}$ are the state vector, input, and output, respectively. The corresponding transfer function is given by $G(z)=C(z I-A)^{-1} B+D$ and $G$ is stable iff $\bar{\rho}(A)<1$. Moreover, $s_{i}$ denotes $s$ at the $i$ th iteration.

\section{FINITE-TIME IIC}

In this section, the tracking control problem is introduced, which is motivated by high-speed scanning probe microscopy (SPM) systems. The FT-IIC method is formulated as a solution to this problem and is subjected to rigorous analysis in the next sections.

\section{A. Application Motivation}

In SPM, such as scanning tunneling microscopy and atomic force microscopy, control is crucial to achieve accurate and fast positioning of the probe with respect to the sample [25] and largely determines the quality of the resulting images [29], as illustrated in Fig. 1. In typical SPM applications, the probe or sample is required to perform a periodic scanning motion. Commonly, the mechanical resonance frequencies of the positioning stage are in the same range as the desired scan rate, such that achieving an acceptable bandwidth using traditional feedback approaches is challenging [25], [30]. Furthermore, the system dynamics may vary due to variations in a sample's size and weight.

\section{B. Problem Formulation}

In view of the control challenges in SPM, it is desired that the developed method satisfies the following requirements.

R1) Periodic references are accurately tracked, and unknown periodic disturbances are rejected.

R2) Convergence is achieved despite plant uncertainties. 


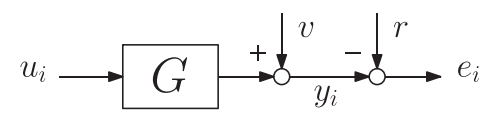

Fig. 2. Control configuration with exogenous output disturbances.

R3) A parametric model of the system is not required.

R4) No additional measures are required to control systems with NMP zeros.

Motivated by the application to SPM, the following assumptions are made.

A1) The process $G$ is a stable LTI system with output disturbance $v(t)$, such that $y_{i}(t)=G(z) u_{i}(t)+v(t)$, as shown in Fig. 2.

A2) The reference $r$ is $N$-periodic and bounded.

A3) The number of samples per period $N$ is known.

Despite the hysteresis that is induced by piezo-based actuation in SPM applications [21], Assumption A1 is commonly made [25] and is especially accurate when a stabilizing low-bandwidth feedback controller is employed that partly linearizes the system, as briefly discussed in Section VI.

The problem of iteratively improving the tracking performance of periodic references is formulated as follows.

Problem 1: Given an $N$-periodic reference $r(t)$, define

$$
e_{i}(t) \triangleq r(t)-y_{i}(t)
$$

where $y_{i}(t)$ is as given in Assumption A1, and where $G(z)$ is given by (1). Determine the $N$-periodic input $u_{i}(t)$ such that $\lim _{i \rightarrow \infty} e_{i}(t)$ is small in an appropriate sense.

In the next section, a frequency-domain approach is proposed as a solution to this periodic tracking problem.

\section{Finite-Time IIC}

The following update law represents the proposed FT-IIC method, which aims to provide a solution to Problem 1 using an FRF model of the process.

Proposition 1: Given an initial input $U_{0}(k)$, update $U_{i}(k)$ as,

$$
U_{i+1}(k)=Q(k)\left(U_{i}(k)+\alpha(k) \hat{G}^{-1}(k) E_{i}(k)\right) \quad \forall k
$$

where $\hat{G}(k)$ is an FRF model of $G\left(e^{j \omega_{k}}\right)$ that satisfies $\hat{G}(k) \neq 0$ $\forall k$, and $Q(k) \in \mathbb{R}_{\geq 0}$ and $\alpha(k) \in \mathbb{R}_{\geq 0}$ are referred to as the robustness and learning coefficient, respectively.

The key attribute of FT-IIC is the explicit use of the DFT to update the input. This enables the use of the nonparametric FRF model $\hat{G}(k)$ and ensures satisfaction of requirement R3. This is essentially different from typical ILC approaches, in which the update is performed by filtering the time-domain signals [6]. The FRF model $\hat{G}(k)$ is typically considered to be a reliable approximation of the plant, and its accuracy can be quantified using various techniques [31]. The coefficients $Q(k)$ and $\alpha(k)$ are used to enable robust convergence and to regulate the learning speed. These coefficients can be tuned separately for each frequency by assuming that the output is approximately in the steady state, since, if $Y(k) \approx G\left(e^{j \omega_{k}}\right) U(k)$, the evolution of $U_{i}(k)$ for frequency $k$ is independent of all other frequencies.

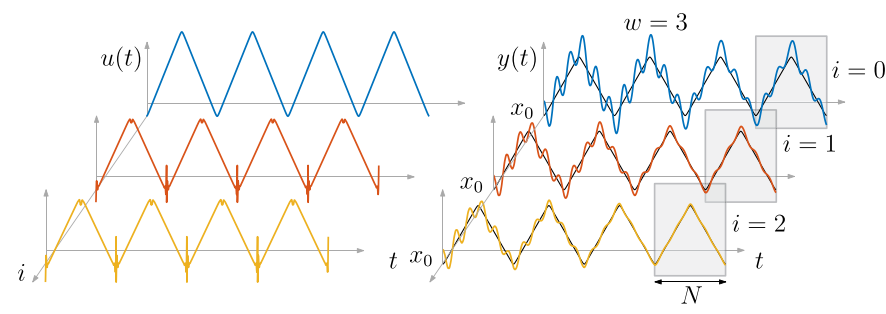

Fig. 3. In the batch-to-batch setting, each task starts from the same initial condition $x_{0}$. After $w$ periods, a single period of $N$ samples is measured when the system is approximately in the steady state (gray intervals). The periodic input is updated based on the measured error.

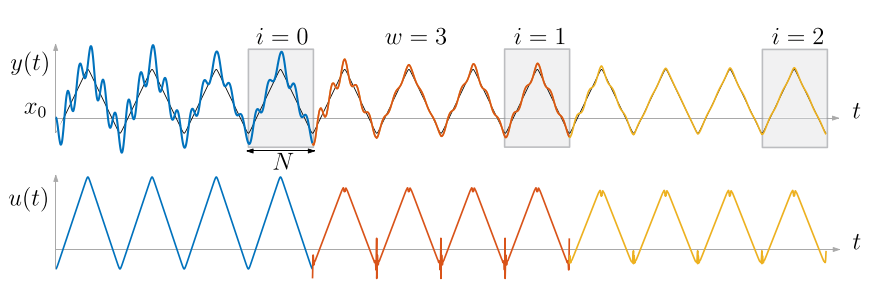

Fig. 4. In the continuous setting, the system starts from initial condition $x_{0}$, which is not reset between updates. After $w$ periods, a single period of $N$ samples is measured when the system is approximately in the steady state (gray intervals). The periodic input is updated based on the measured error.

This approach is enabled by the following implementation framework, where the distinction between batch-to-batch and continuous updating is made explicit in Section V-A and Figs. 3 and 4.

Remark 1: Since the DFT is an integral part of FT-IIC, the results in this paper apply to $N$-periodic and time-limited signals [28]. Time-limited tasks are naturally implemented in a batchto-batch fashion with $w=0$, which removes the freedom to wait for the steady state. Furthermore, for $N \rightarrow \infty$, the classical IIC and infinite-time ILC methods are reobtained.

\section{Outline}

The remainder of this paper is structured as follows. In light of requirement R4, it is shown in Section III that (3) results in bounded inputs, even when $G(z)$ is NMP. In Section IV, the convergence behavior and asymptotic properties of (3) are derived under the assumption that the system is in the steady state, which results in tuning guidelines for $Q(k)$ and $\alpha(k)$ to satisfy requirements R1 and R2. In Section V, finite-time effects are explicitly analyzed by means of a lifted signal framework, which results in conditions on the number of waiting periods $w$ to ensure convergence. In Section VI, the developed FT-IIC method is applied to a nanopositioning stage.

\section{Fourier-Transform-BASEd StABLE INVERSION}

In this section, it is shown that in contrast to inverse-modelbased learning, the presented inverse-FRF-based approach results in bounded signals, irrespective of the potential presence of NMP dynamics. This is shown by explicitly solving the stable inversion problem in the frequency domain, which constitutes 


Algorithm 1: FT-IIC.
Given an initial periodic input $u_{0}(t)$.
1) Batch-to-batch: reset the system to a certain initial $x_{0}$.
Continuous: do not reset the system.
2) Apply the $N$-periodic input $u_{i}(t)$ to the system and wait
$w$ periods until the output $y_{i}(t)$ is approximately in the
steady state. Then, record the tracking error $e_{i}(t)(2)$.
3) a) Obtain $U_{i}(k)$ and $E_{i}(k)$ by applying the DFT.
b) Update $U_{i}$ by means of (3) for each frequency $k$.
c) Obtain $u_{i+1}(t)$ by applying the IDFT.
4) Set $i \leftarrow i+1$ and repeat from step 1$)$ until $e_{i}(t)$ converges.

contribution $\mathrm{C} 1$. More specifically, the aim of this section is to derive conditions under which

$$
u(t)=\mathcal{F}_{N}^{-1}\left\{G^{-1}(k) \mathcal{F}_{N}\{r(t)\}\right\}
$$

is guaranteed to be bounded while satisfying $y(t)=r(t)$.

\section{A. Stable Inversion in the Time Domain}

The stable inversion problem is first considered in the time domain. Considering (2) for $v(t)=0$ shows that the perfect tracking input is obtained by inverting the relation $G u=r$. Ensuring that $u(t)$ is bounded is known as the problem of stable inversion, which is formulated as follows [14].

Problem 2 (Stable inversion): Let $G: u \mapsto y$ be a DT-LTI system. Then, for a certain $r \in \ell_{\infty}$, determine $u \in \ell_{\infty}$ such that (1) holds in addition to $y(t)=r(t) \forall t \in \mathbb{Z}$.

A time-domain solution [14] follows by inverting the statespace realization of $G$, i.e., (1), which is given by

$$
G^{-1}:\left[\begin{array}{c|c}
\tilde{A} & \tilde{B} \\
\hline \tilde{C} & \tilde{D}
\end{array}\right]=\left[\begin{array}{c|c|c}
A-B D^{-1} C & B D^{-1} \\
\hline-D^{-1} C & D^{-1}
\end{array}\right] .
$$

Note that this can be extended to the case with $D=0$ by using input or output previewing [32]. When $G$ is NMP, at least one of the zeros of $G(z)$ lies outside of the unit circle. This renders $G^{-1}$ unstable in a causal sense, since the zeros of $G$ are equal to the poles of $G^{-1}$. If none of the eigenvalues of $\tilde{A}$ lies on the unit circle, a similarity transformation $\eta=T \tilde{x}$ can be performed that decouples the dynamics in a stable and antistable part [14]. This dichotomic split results in

$$
G^{-1}:\left\{\begin{aligned}
{\left[\begin{array}{l}
\eta_{s}(t+1) \\
\eta_{u}(t+1)
\end{array}\right] } & =\left[\begin{array}{cc}
\tilde{A}_{s} & 0 \\
0 & \tilde{A}_{u}
\end{array}\right]\left[\begin{array}{l}
\eta_{s}(t) \\
\eta_{u}(t)
\end{array}\right]+\left[\begin{array}{c}
\tilde{B}_{s} \\
\tilde{B}_{u}
\end{array}\right] y(t) \\
u(t) & =\left[\begin{array}{ll}
\tilde{C}_{s} & \tilde{C}_{u}
\end{array}\right] \eta(t)+\tilde{D} y(t) .
\end{aligned}\right.
$$

A bounded yet noncausal $u$ is obtained by iterating $\eta_{s}$ forward in time and $\eta_{u}$ backward in time for $y=r$, i.e.,

$$
\eta_{s}(t)=\sum_{i=-\infty}^{t-1} \tilde{A}_{s}^{t-i-1} B_{s} y(i), \quad \eta_{u}(t)=-\sum_{i=t}^{\infty} \tilde{A}_{u}^{t-i-1} B_{u} y(i) .
$$

This approach solves Problem 2 if $G$ has neither zeros nor poles on $\mathbb{C}^{\circ}$ [14]. Notably, this time-domain approach requires a state-space model of the system. Next, a frequency-domain approach is developed that only requires an FRF model.

\section{B. Stable Inversion in the Frequency Domain}

In this section, Problem 2 is solved by using a Fourier transform approach that does not explicitly use a parametric model. The key aspect in this approach is that boundedness is guaranteed if the Fourier transforms are well defined.

Lemma 1: Let $G$ be a stable system satisfying (1). If for a certain $r, R(\omega)=\mathcal{F}_{\infty}\{r(t)\}$ exists, and if $G\left(e^{j \omega}\right)^{-1} R(\omega)$ is bounded $\forall \omega \in(-\pi, \pi]$, then

$$
u=\mathcal{F}_{\infty}^{-1}\left\{\left[G\left(e^{j \omega}\right)\right]^{-1} \mathcal{F}_{\infty}\{r(t)\}\right\}
$$

is such that $u \in l_{\infty}$, and (1) holds with $y(k)=r(k) \forall k \in \mathbb{Z}$.

A proof of this lemma is provided in Appendix A. This lemma shows that the DTFT-based operation (4) solves Problem 2 and only requires the FRF $G\left(e^{j \omega}\right)$. Note that $G\left(e^{j \omega}\right)^{-1} R(\omega)$ is bounded if $G(z)$ has no zeros on $\mathbb{C}^{\circ}$, yet this does not allow for cancellations between $G\left(e^{j \omega}\right)^{-1}$ and $R(\omega)$. Furthermore, the requirement that $\mathcal{F}_{\infty}\{r\}$ exists can be relaxed by adopting the notion of generalized DTFTs [14].

Remark 2: In Lemma 1, $u(t)$ is noncausal if $G$ is NMP. This is shown by considering $G(z)$ as the bilateral Z-transform [28, Sec. 2.13] of the impulse response of $G$, and $G^{-1}(z)$ as the $z$ componentwise inverse of $G(z)$. A bounded impulse response of $G^{-1}(z)$ is obtained by applying the inverse bilateral Z-transform over the contour $z=e^{j \omega}$, such that the region of convergence includes $\mathbb{C}^{\circ}$. Since $G(z)$ has no zeros on $\mathbb{C}^{\circ}$, this results in a bounded impulse response, which is noncausal if $G(z)$ possesses zeros outside the unit disc [28, Sec. 2.13.2].

In conclusion, the DTFT can be used to solve the stable inversion problem by using a continuous FRF model. Next, the DFT is considered to enable the use of a discrete FRF.

\section{Periodic Steady-State Stable Inversion}

To apply the results of Lemma 1 to finite-time signals, the DTFT is replaced by the DFT on a finite grid of frequencies. Truncating the infinite-time solution is exact if the involved signals are periodically repeating outside the considered time interval [28, Sec. 2.21]. Consequently, (4) can be applied by using the DFT to obtain the periodic input sequence that leads to a given desired periodic output, even if the system is NMP.

Theorem 1: Let $G$ be a stable system satisfying (1) with FRF $G\left(e^{j \omega_{k}}\right)=C\left(e^{j \omega_{k}} I-A\right)^{-1} B+D, \omega_{k} \in \Omega$,

$$
\Omega \triangleq\left\{\omega_{k} \in \mathbb{R} \mid \omega_{k}=\frac{2 \pi}{N} k, k=0, \ldots, N-1\right\} .
$$

If for a certain $N$-periodic $r, R(k)=\mathcal{F}_{N}\{r(t)\}$ exists, and if $G\left(e^{j \omega_{k}}\right)^{-1} R(k)$ is bounded $\forall \omega_{k} \in \Omega$, then

$$
u(t)=\mathcal{F}_{N}^{-1}\left\{\left[G\left(e^{j \omega_{k}}\right)\right]^{-1} \mathcal{F}_{N}\{r(t)\}\right\}
$$

is such that $u \in l_{\infty}$, and (1) holds in addition to $y(k)=r(k)$ $k \in 0, \ldots, N-1$, where $u$ and $y$ are $N$-periodic.

Proof: With the fact that $\tilde{x}(k)=\mathcal{F}_{N}^{-1}\left\{\mathcal{F}_{N}\{x\}\right\}=x(k)$ $\forall k \in \mathbb{Z}$ if $x$ is $N$-periodic [28, Sec. 2.21], a proof follows identically as the proof of Lemma 1 by using $\mathcal{F}_{N}$ instead of $\mathcal{F}_{\infty}$, and by restricting $u, x$ and $y$ to be $N$-periodic signals.

This theorem states that the bounded $N$-periodic input $u$ that results in the tracking of an $N$-periodic reference $r$ can be 
computed by using an FRF model of $G$ that is defined on the DFT grid $\Omega(5)$. Note that this requires that $G$ is in the steady state, i.e., the state $x$ is $N$-periodic.

In this section, the theoretical motivation for FT-IIC (3) is provided by developing a frequency-domain approach to the stable inversion problem. It is shown that bounded and possibly noncausal solutions are automatically obtained by using an FRF model in combination with the DFT. Consequently, FT-IIC satisfies requirement R4. In the next section, FT-IIC is analyzed in the frequency domain.

\section{Steady-State ANALYSis}

In this section, the convergence behavior and asymptotic properties of (3) are derived. These results lead to guidelines to tune $Q(k)$ and $\alpha(k)$, which constitutes contribution C2.

In the remainder of this section, it is assumed that the $N$ periodic steady-state output of $G$ is measured, such that

$$
Y_{i}(k)=G\left(e^{j \omega_{k}}\right) U_{i}(k)+V(k)
$$

where $V$ is a bounded trial-invariant disturbance, i.e., $V_{i}=V$ $\forall i$. Furthermore, the dependence on $k$ is occasionally omitted to facilitate a clear exposition.

\section{A. Optimal FT-IIC}

The FT-IIC update law, as given by (3), is shown to connect to a regularized least squares optimization problem that is commonly posed in norm-optimal ILC [2]. This reveals the particular roles of $Q(k)$ and $\alpha(k)$.

Lemma 2: Consider the following optimization problem:

$$
\begin{aligned}
U_{i+1}(k) & =\arg \min \mathcal{J}(k) \quad \forall k \\
\mathcal{J} & =\left|\hat{E}_{i+1}\right|^{2}+w_{u}\left|U_{i+1}\right|^{2}+w_{\Delta u}\left|U_{i+1}-U_{i}\right|^{2}
\end{aligned}
$$

where $w_{u}(k), w_{\Delta u}(k) \geq 0$ are weights and $\hat{E}_{i} \triangleq R-\hat{G} U_{i}-$ $V$, where $\hat{G}$ is an FRF model of $G$. The FT-IIC update law given by (3) is the solution of this optimization problem if

$$
Q=\frac{|\hat{G}|^{2}+w_{\Delta u}}{|\hat{G}|^{2}+w_{\Delta u}+w_{u}}, \quad \alpha=\frac{|\hat{G}|^{2}}{|\hat{G}|^{2}+w_{\Delta u}} .
$$

A proof of this lemma is provided in Appendix B. Considering (7) for $w_{\Delta_{u}}=0$ shows that $\alpha(k)=1$ corresponds to the case for which the rate of change of $U_{i}(k)$ is not restricted from one trial to the next. Similarly, taking $w_{\Delta}=0$ shows that $Q(k)=1$ corresponds to the case for which $\left|U_{i+1}(k)\right|$ is not restricted. In the next sections, additional frequency-domain tuning design guidelines are derived for $Q(k)$ and $\alpha(k)$ to achieve disturbance rejection, monotonic convergence, and robustness against plant variations, respectively.

\section{B. Disturbance Rejection}

High performance is achieved if the tracking error is sufficiently small. If the input updating (3) converges, the resulting asymptotic tracking error is as follows.
Lemma 3: If the FT-IIC update, as given by (3), converges, then the asymptotic error is given by

$$
E_{\infty}(k)=\frac{1-Q(k)}{1-Q(k)\left(1-\alpha(k) G\left(e^{j \omega}\right) \hat{G}^{-1}(k)\right)} R_{v}(k)
$$

where $R_{v} \triangleq R-V$ is the total periodic disturbance.

A proof of this lemma is provided in Appendix C. From (8), it is clear that perfect rejection is obtained for $Q(k)=1$. Consequently, requirement $\mathrm{R} 1$ is satisfied, as long as convergence is achieved with $Q(k)=1$. Next, it is shown that this can be achieved if the FRF model is sufficiently accurate.

\section{Monotonic Convergence}

To avoid bad learning transients [6], [33], it is key that $Q(k)$ and $\alpha(k)$ are chosen such that (3) results in monotonically converging sequences, which are defined as follows.

Definition 1: A sequence $\left\{X_{i}\right\}, X \in \mathbb{C}$, is said to convergence monotonically in the absolute value to a fixed point $X_{\infty} \in \mathbb{C}$ if there exists a $\kappa \in[0,1)$ such that

$$
\left|X_{i+1}-X_{\infty}\right| \leq \kappa\left|X_{i}-X_{\infty}\right| \quad \forall i
$$

where $\kappa^{-1}$ is referred to as the rate of convergence.

The following theorem provides a sufficient condition such that the FT-IIC input sequences converge monotonically.

Lemma 4: The sequence $\left\{U_{i}(k)\right\}$ resulting from (3) converges monotonically at frequency $k$, at a rate of $\kappa^{-1}(k)$, if

$$
\kappa(k) \triangleq\left|Q(k)\left(1-\alpha(k) \hat{G}^{-1}(k) G\left(e^{j \omega}\right)\right)\right|<1 .
$$

A proof to this lemma is provided in Appendix D. From (9), it is clear that both $Q(k)$ and $\alpha(k)$ can be used to achieve monotonic convergence. Note that in IIC [19], $Q(k)=1$ such that only $\alpha(k)$ can be used to achieve convergence. In the next section, it is shown that by taking $Q(k) \neq 1$, performance can be exchanged for robustness against plant variations, which is a mechanism that is lacking in pre-existing IIC approaches.

\section{Robust Convergence}

The requirement of robust convergence, $\mathrm{R} 2$, can be analyzed by considering the following additive uncertain model set:

$$
\begin{aligned}
\mathcal{G}(\Delta, k) & =\hat{G}(k)+\Delta(k), \quad \Delta(k) \in \boldsymbol{\Delta} \\
\boldsymbol{\Delta} & \triangleq\left\{\Delta \in \mathbb{C}|| \Delta \mid \leq \delta, \delta \in \mathbb{R}_{\geq 0}\right\}
\end{aligned}
$$

where $\hat{G}$ is the nominal model and $\Delta$ is a bounded uncertainty. Such a description can be estimated by using FRF identification methods [31, Sec. 7.2.4]. By assuming that the true FRF is captured by the model set, i.e., $\exists \Delta^{*} \in \Delta, G=\mathcal{G}\left(\Delta^{*}\right)$, robust convergence can be analyzed by substituting (10) into (9)

$$
\begin{aligned}
& \kappa(\Delta, k)=|Q(k)(1-\alpha(k) \varphi(k))|<1 \\
& \varphi(\Delta, k)=1+\hat{G}^{-1}(k) \Delta(k) .
\end{aligned}
$$

More specifically, (3) is said to converge robustly with respect to (10), if (11) is satisfied $\forall \Delta \in \Delta$. In the case of perfect tracking, i.e., for $Q=1$, then (11) can only be satisfied if $\varphi(\Delta) \in \mathbb{C}_{>0}$, 
since by definition, $\alpha \geq 0$. If convergence is not achieved with respect to the true system, i.e., $\varphi\left(\Delta^{*}\right) \notin \mathbb{C}_{>0}$, then convergence is achieved by adjusting the phase of the nominal model by $180^{\circ}$, i.e., $-\hat{G}^{-1}(k)$, since $\varphi\left(\Delta^{*}\right) \in \mathbb{C}_{>0}$ in this case. However, if $\left|\varphi\left(\Delta^{*}\right)\right| \gg 1$, (11) requires that $\alpha \ll 1$, and consequently, $\kappa \approx$ 1 , which implies slow convergence. This is alleviated by taking $Q(k)<1$, which increases robustness, at the cost of perfect tracking. The following lemma provides the key to achieving robust convergence by suitable design of $Q$ and $\alpha$.

Lemma 5: If $\exists \Delta^{*} \in \Delta$ such that $G=\mathcal{G}\left(\Delta^{*}\right)$ and if $\alpha(k) \in$ $(0,1]$, then condition (11) is independent of $\alpha(k)$. Furthermore, if $\alpha(k)=1$, then (11) is satisfied $\forall \Delta \in \Delta$ if

$$
\left\{\begin{array}{l}
Q(k)=1, \quad \text { for } \tau(k)>1 \\
Q(k)<\tau, \quad \text { for } \tau(k) \leq 1
\end{array}, \quad \tau(k) \triangleq \frac{|\hat{G}(k)|}{\delta(k)} .\right.
$$

A proof of this lemma is provided in Appendix E. In this section, it is shown that robust convergence is achieved by proper choice of $Q(k)$ and $\alpha(k)$. Next, these results are reformulated into design guidelines.

\section{E. Design Guidelines}

Based on the developed insights, design guidelines are formulated to achieve high performance and robust convergence such that requirements R1 and R2 are satisfied by FT-IIC.

The following guideline presents an approach to maximize the performance in a trial-and-error fashion in case only a nominal FRF model is available.

Design Guideline 1: Given a nominal FRF model $\hat{G}(k)$ :

1) set $Q(k)=1$, and through preliminary experiments, set $\alpha(k) \in(0,1] \forall k$ as high as possible such that the input converges to a signal that is approximately identical for each trial as opposed to increasing without bounds;

2 ) if convergence is not achieved for some $k$, change the phase of $\Delta(k)$ by setting $\alpha(k) \hat{G}^{-1}(k) \rightarrow-\alpha(k) \hat{G}^{-1}(k)$ and repeat the above;

$3)$ in case $|\alpha(k)| \ll 1$ for some $k$, set $Q(k)<1$ to increase the convergence speed and robustness.

The next guideline summarizes the approach in case robustness against plant variations is critical, or in case a trial-and-error approach is unacceptable. This requires an additive uncertain description as given by (10).

Design Guideline 2: Given a nominal FRF model $\hat{G}(k)$ and additive uncertainty $\Delta(k)$ such that (10) holds, set $Q(k)$ as given by (12) and set $\alpha(k)=1 \forall k$.

In this section, the convergence behavior and asymptotic properties of (3) are derived. This results in design guidelines for $Q(k)$ and $\alpha(k)$, thereby providing an FT-IIC framework, as proposed in Section II, that satisfies the posed requirements.

In the next section, the effect of the transient response is analyzed in the time domain.

\section{FINITE-TIME ANALYSIS}

In the previous sections, it is assumed that the output is measured during the steady state. In practice, there is a contribution of the transient response that results from starting up the system, or from updating of the periodic input signal, which decays as the systems operates. In this section, the effects of transients are explicitly analyzed with respect to the convergence of FT-IIC. This results in conditions on the number of waiting periods $w$ that are required to achieve convergence in the presence of transients, which constitutes contribution C3.

\section{A. Finite-Time Behavior}

If the input to $G$ is periodic, then the output converges to a periodic signal. Depending on the pole locations, the steady state is approximately obtained after some periods $w$. Hence, FT-IIC can be applied in a setting that allows for some waiting time between the input updating. In this section, two setting are considered. The first is the batch-to-batch setting, where the system resets after each update, as shown in Fig. 3, for $w=3$. The second is the continuous setting, where the system operates without resetting, as shown in Fig. 4.

To analyze the transient behavior, a lifted signal description is employed that enables the consideration of an entire period at once. For a signal $s(t)$, the corresponding lifted signal $\bar{s}^{\langle n\rangle}$ is given by grouping $N$ consecutive samples of the signal

$\bar{s}^{\langle n\rangle} \triangleq[s(N n) s(N n+1) \ldots s(N n+N-1)]^{\top}, \quad n \in \mathbb{Z}$.

For $N$-periodic signals, it holds that $\bar{s}^{\langle n\rangle}=\bar{s} \forall n$, since $s(t)=$ $s(t+N) \forall t$. By lifting $u(t)$ and $y(t)$ of $G$, as given by (1), a lifted system $G_{l}: \bar{u}^{\langle n\rangle} \mapsto \bar{y}^{\langle n\rangle}$ is obtained, whose realization follows by evaluating (1a), (1b), and $x(0)=x_{0}$ as in [34]

$$
\begin{aligned}
& G_{l}: \begin{cases}x(N(n+1)) & =F x(N n)+M \bar{u}^{\langle n\rangle} \\
\bar{y}^{\langle n\rangle} & =H x(N n)+J \bar{u}^{\langle n\rangle} \\
x(0) & =x_{0}\end{cases} \\
& {\left[\begin{array}{c|c}
F \mid M \\
\hline H \mid J
\end{array}\right]=\left[\begin{array}{c|cccc}
A^{N} & A^{N-1} B & \ldots & A B & B \\
\hline C & h(0) & 0 & \ldots & 0 \\
C A & h(1) & h(0) & & 0 \\
\vdots & \vdots & \ddots & \ddots & \vdots \\
C A^{N-1} & h(N-1) & \ldots & h(1) & h(0)
\end{array}\right]}
\end{aligned}
$$

where $h(t)$ are the Markov parameters, i.e., $h(t)=D$ for $t=$ 0 and $h(t)=C A^{t-1} B$ for $t \geq 1$. This framework allows the analysis of FT-IIC in the time domain, as treated next.

\section{B. FT-IIC in the Time Domain}

A number of auxiliary results are derived that enable the transient analysis of FT-IIC in the next section. First, the FT-IIC update law (3) is formulated in the lifted time domain. This requires lifting of the DFT operation, which is facilitated by the DFT matrix $\mathcal{F} \in \mathbb{C}^{N \times N}$, i.e., $[\mathcal{F}]_{h k}=\frac{1}{\sqrt{N}} \mu^{h k}$ for $h, k=$ $0, \ldots, N-1$, where $\mu=e^{-\frac{2 \pi j}{N}}[35$, Sec. 1.4.1]. It follows from the definition of the DFT that $\bar{S}=\mathcal{F} \bar{s}$, where $\bar{S}(k)$ results from lifting $S(k)$. Furthermore, it holds that $\bar{s}=\mathcal{F}^{H} \bar{S}$, since $\mathcal{F}$ is a unitary matrix [35, Sec. 4.8.1]. This formulation enables the 
connection of the lifted frequency-domain steady-state behavior to the lifted time domain.

Lemma 6: Given a lifted system $G_{l}$ as given by (13). If the state is periodic, i.e., if $x(N(n+1))=x(N n)$, then $\bar{y}=J_{p} \bar{u}$, where $J_{p}$ is the periodic response matrix

$$
J_{p} \triangleq H(I-F)^{-1} M+J .
$$

Furthermore, $J_{p}$ is equivalent to

$$
J_{p}=\mathcal{F}^{H} \operatorname{diag}\left\{G\left(e^{j \omega_{k}}\right)\right\} \mathcal{F}
$$

where $\mathcal{F}$ is the DFT matrix and $G\left(e^{j \omega_{k}}\right)$ the FRF of $G$.

A proof of this lemma is provided in Appendix F. This lemma enables the formulation of the FT-IIC update law (3) in the lifted time domain.

Lemma 7: A lifted representation of (3) is given by

$$
\begin{aligned}
\bar{u}_{i+1} & =\mathcal{Q}\left(\bar{u}_{i}+\Lambda \hat{J}_{p}^{-1} \bar{e}_{i}\right) \\
\mathcal{Q} & \triangleq \mathcal{F}^{H} \operatorname{diag}\{Q(k)\} \mathcal{F}, \quad \Lambda \triangleq \mathcal{F}^{H} \operatorname{diag}\{\alpha(k)\} \mathcal{F}
\end{aligned}
$$

$k=0, \ldots, N-1$, where $\hat{J}_{p}$ corresponds to the FRF model $\hat{G}(k)$ and is given by Lemma 6 , and $\mathcal{F}$ is the DFT matrix.

Proof of Lemma 7: Lifting (3) results in

$$
\bar{U}_{i+1}=\operatorname{diag}\{Q(k)\}\left(\bar{U}_{i}+\operatorname{diag}\left\{\alpha(k) G_{m}^{-1}(k)\right\} \bar{E}_{i}\right) .
$$

Using that $\bar{S}=\mathcal{F} \bar{s}$ and $\mathcal{F}^{H} \bar{S}=\bar{s}$ in combination with Lemma 6 directly results in (16).

By property of $\mathcal{F}$, the matrices $\mathcal{Q}, \Lambda$, and $J_{p}$ are circulant [35, Sec. 4.8.2] and, thus, correspond to time-invariant and noncausal linear mappings, as was similarly discussed in Remark 2. Furthermore, note that $\hat{J}_{p}$ is invertible if $\hat{G}(k) \neq 0 \forall k$, which is in agreement with Theorem 1.

As established in Section V-A, the output used in the update can be obtained by waiting until the steady state is approximately attained. To analyze the effect of remaining transients, consider the following explicit expression of the output during the $w$ th period since the input was last updated.

Lemma 8: Given $G_{l}$ as given by (13). If $G_{l}$ is excited by an $N$-periodic input $\bar{u}$, then the lifted output during the $w$ th period is given by

$$
\begin{aligned}
\bar{y}^{\langle n+w\rangle} & =H F^{w} x(N n)+J_{t} \bar{u}^{\langle n\rangle} \\
J_{t} & \triangleq H \sum_{j=0}^{w-1} F^{j} M+J .
\end{aligned}
$$

Proof: A proof follows directly by forward recursion of (13) with a periodic input $\bar{u}_{i}$.

In this section, lemmas are presented that facilitate a timedomain convergence analysis of FT-IIC, as treated next.

\section{Time-Domain Convergence Analysis}

Combining the auxiliary lemmas enables the main result of this section, which considers a nonconservative convergence analysis for both the batch-to-batch and continuous setting.
Theorem 2: Let $G$ be a stable system satisfying (1) and consider the tracking error given by (2). If FT-IIC is used to iteratively update the input as given by (16), where the output is measured after waiting $w$ periods, then the following holds.

1) Batch-to-batch: If updating is performed in a batch-tobatch fashion, as shown in Fig. 3 , then $\bar{u}_{i}$ converges if and only if $\bar{\rho}(Z)<1$ and converges monotonically in the 2 -norm if $\bar{\sigma}(Z)<1$, with $J_{t}$ given by (18) and

$$
Z=\mathcal{Q}\left(I-\Lambda \hat{J}_{p}^{-1} J_{t}\right)
$$

2) Continuous: If updating is performed in a continuous fashion, as shown in Fig. 4, then the closed-loop system is asymptotically stable if and only if $\bar{\rho}(\mathcal{A})<1$, with

$$
\mathcal{A}=\left[\begin{array}{cc}
F^{w} & \sum_{j=0}^{w-1} F^{j} M \\
-\mathcal{Q} \Lambda \hat{J}_{p}^{-1} H F^{w} & Z
\end{array}\right] .
$$

Furthermore, if the model is perfect, i.e., $\hat{G}(k)=G\left(e^{j \omega_{k}}\right)$, and if $\alpha(k)=1 \forall k$, then $Z$ is identical to

$$
Z_{\circ}=\mathcal{Q} J_{p}^{-1}\left(H F^{w}(I-F)^{-1} M\right) \text {. }
$$

A proof to this theorem is provided in Appendix G. This theorem provides conditions for the convergence of FT-IIC in the presence of transients. More specifically, in the batch-tobatch setting, stable evolution of $\bar{u}^{\langle n\rangle}$ is governed by $\bar{\rho}(Z)$. Consequently, $\bar{\rho}(Z)<1$ provides a condition on the number of waiting periods $w$ that are required to achieve convergence, which is typically small. To see this, consider the case where the model is perfect, i.e., $Z$ equals $Z_{\circ}$, (20). Using that $\bar{\rho}\left(F^{w}\right)=$ $\bar{\rho}(A)^{N \cdot w}$, where $\bar{\rho}(A)<1$ by assumption, it follows readily that $F$, and therefore $Z_{\circ}$, converges to 0 for moderate $w$ and $N$. This holds similarly for the continuous setting, in which case the matrix $\mathcal{A}$ is considered to also guarantee the stability of the state $x$.

Remark 3: For $w=0$, (17) satisfies $\bar{y}^{\langle n\rangle}=H x(N n)+$ $J \bar{u}^{\langle n\rangle}$. Consequently, the lifted ILC problem is recovered in the batch-to-batch setting, and the lifted RC problem is recovered in the continuous setting [34]. Hence, if $w=0$ and depending on the way in which FT-IIC is implemented, it can be interpreted either as ILC or RC. Comparatively, FT-IIC prescribes the inverse FRF model-based learning matrix $L=\Lambda J_{p}^{-1}$, whereas several alternative methods exist to design this matrix for lifted ILC or RC [36]. Such methods implicitly account for the transient effects, whereas the influence of the transients is mitigated by waiting in FT-IIC, i.e., by taking $w>0$.

Remark 4: In practice, a waiting time consisting of an arbitrary number of samples can be used, as long as the updated periodic input is synchronized with the original input upon application to the system, for example, by using the circular shift operation [28].

In this section, a time-domain analysis of the developed FTIIC is treated, which shows that convergence can be achieved by waiting for an approximate steady state. In the next section, the FT-IIC approach is applied to control a nanopositioner. 


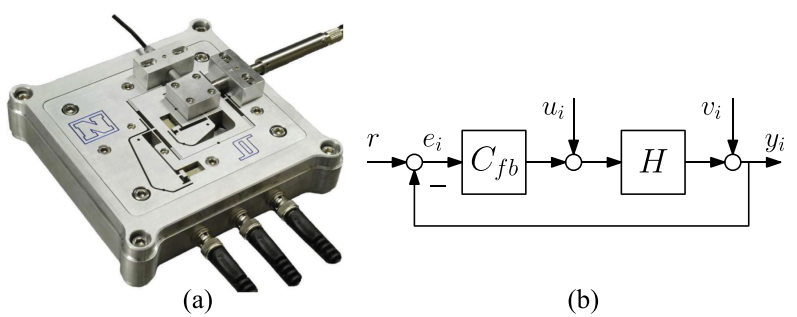

Fig. 5. (a) Three-axis nanopositioner designed by K. K. Leang at the University of Utah. The axes contain a 12-mm-long piezoelectric stack actuator of the type, Noliac NAC2003- H12, with a free displacement of $12 \mu \mathrm{m}$ at $200 \mathrm{~V}$, which is extended by mechanical amplifiers to a total range of $30 \mu \mathrm{m}$. Each actuator is driven by a PiezoDrive PDL200 voltage amplifier. A Microsense 6810 capacitive sensor and 6504-01 probe, with $2.5-\mu \mathrm{m} / \mathrm{V}$ sensitivity, is used to measure the position of the moving platform. Two SRS SIM965 analog filters serve as reconstruction and antialiasing filters, respectively, by configuring these as Butterworth filters with a cutoff frequency of $3 \mathrm{kHz}$ and a slope of $-12 \mathrm{~dB}$. (b) Closedloop control configuration.

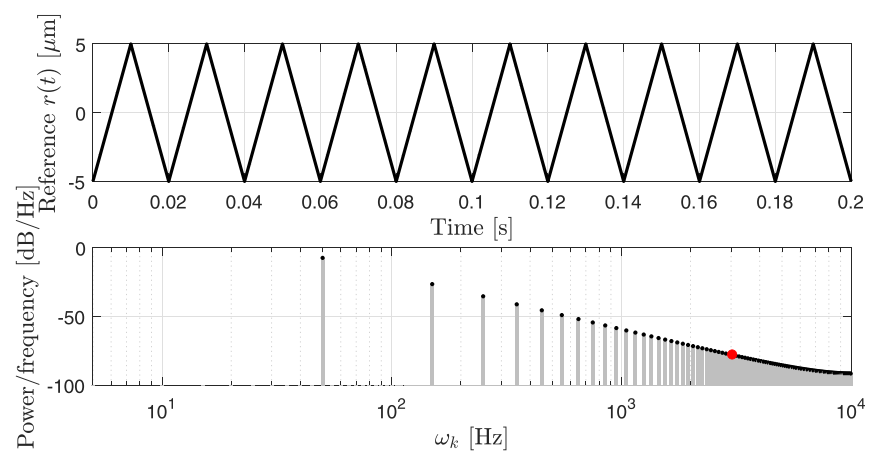

Fig. 6. (Top) Reference $r(t)(-)$. (Bottom) PSD of $r(t)(\bullet)$. The point $(\bullet)$ indicates the contribution at $\omega_{k}^{*}=3050 \mathrm{~Hz}$.

\section{High-Precision Periodic Nanopositioning}

In this section, the potential of the FT-IIC method is demonstrated by achieving precise control of a nanopositioning stage, which constitutes contribution $\mathrm{C} 4$.

\section{A. Control Problem}

The control objective is to perform a periodic scanning motion with the $x$-axis of the nanopositioner shown in Fig. 5(a). A single trial takes $0.2 \mathrm{~s}$ and consists of ten periods of a triangle wave with an amplitude of $5 \mu \mathrm{m}$, shown in Fig. 6. A feedback controller is used to provide a benchmark level of performance and is given by

$$
C_{f b}(z)=\frac{6.1078 \cdot 10^{-6}(z+1)^{4}(z-0.9844)}{(z-0.9099)^{2}(z-0.975)(z-1)(z-0.7007)}
$$

which achieves a bandwidth of $90 \mathrm{~Hz}$ and is operated at a sampling frequency of $20 \mathrm{kHz}$. The FT-IIC method is applied to update the feedforward signal $u_{i}$, as shown in Fig. 5(b).

\section{B. Implementation}

The FT-IIC method is implemented in a continuous setting, as presented in Section II and as shown in Fig. 4. The system is
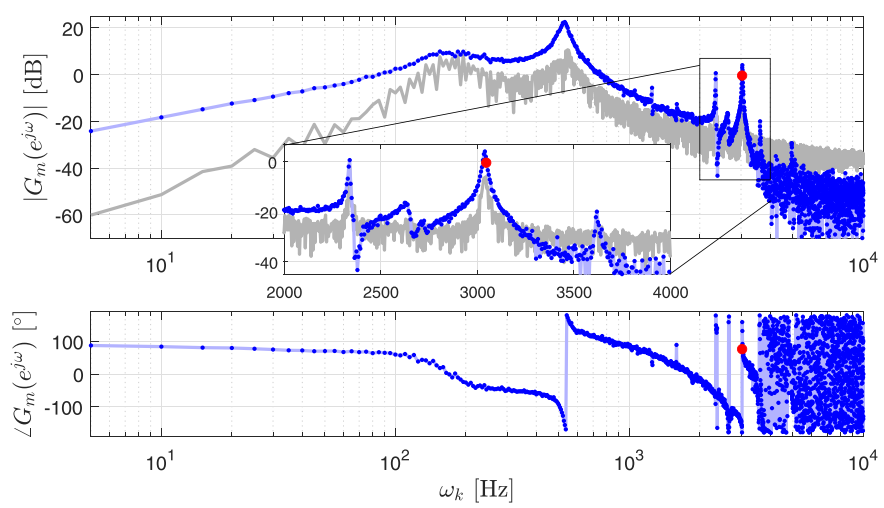

Fig. 7. Bode plot of the $\operatorname{FRF} \hat{G}(k)(\bullet)$, and a first-order estimate of its standard deviation (-). The point $(\bullet)$ indicates the FRF at $\omega_{k}^{*}=3050$ $\mathrm{Hz}$.

first brought into continuous operation by means of the feedback controller. At the end of each trial, a trigger indicates that data acquisition can start. In this way, sequences of exactly $N=$ 4000 samples are obtained. After initialization, the input $u_{i}$ is iteratively updated after three periods. The first period is used for the system to settle to the steady state, i.e., $w=1$. During the second period, $e_{i}$ is measured. The computation of $u_{i+1}$ is performed during the third period by using the update law (3), wherein the DFT operation and its inverse are computed using the fast Fourier transform algorithm. Optimal time-distributed transform algorithms facilitate computation within one sample period [37], but are beyond the scope of the current exposition.

\section{Application of FT-IIC}

In the closed-loop configuration, $G$ is the process sensitivity. The FRF estimate $\hat{G}(k)=\hat{H}(k)\left(1+\hat{H}(k) C_{f b}\left(e^{j \omega_{k}}\right)\right)^{-1}$ is constructed by using an estimate of the process $\hat{H}$. An H1 estimator of $H(k)$ is obtained by exciting the system with a full spectrum periodic input in open loop, and by dividing the average cross power spectrum density (PSD) by the average auto PSD [31]. The resulting model $\hat{G}(k)$ and a first-order approximation of its standard deviation are shown in Fig. 7. This shows that for frequencies $>3 \mathrm{kHz}$, the uncertainty is relatively large. Furthermore, $\hat{G}^{-1}(k)$ is readily obtained for each $k$ with exception of $k=0$, since $\hat{G}(0)=0$ due to the integrator in the feedback controller. To satisfy the condition in Theorem 1, $\hat{G}^{-1}(0)$ is set to zero.

To illustrate that high performance can be achieved with low requirements on prior modeling, Design Guideline 1 is used, since it does not require an uncertainty model. As described in step $1, Q(k)=1 \forall k$, and $\alpha(k)$ is set to $\alpha_{c}=0.6 \forall k$ to allow convergence over a large frequency range. After a first exploratory experiment, it turns out that convergence is achieved up to $3700 \mathrm{~Hz}$, with exception of a small set of frequencies, including $\omega_{k}^{*}=3050 \mathrm{~Hz}$. At these nonconvergent frequencies, $\alpha(k)$ is set to 0, as shown in Fig. 8. Conversely, in the lower frequency range, $\alpha(k)$ can potentially be increased toward 1 at certain frequencies, which typically increases the convergence speed. 


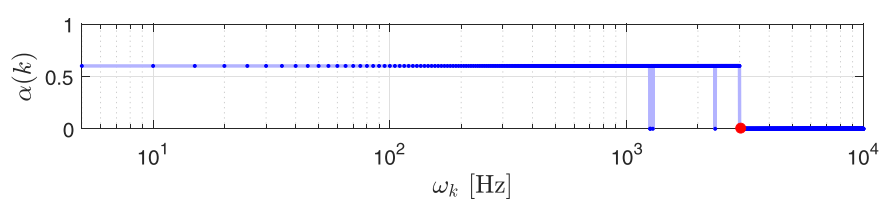

Fig. 8. Learning coefficient $\alpha(k)(\bullet)$. The point $(\bullet)$ indicates the learning coefficient at $\omega_{k}^{*}=3050 \mathrm{~Hz}$, which is zero.

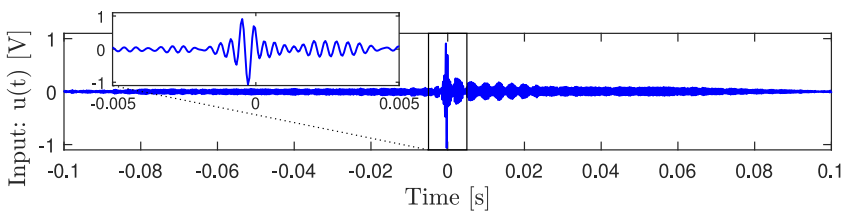

Fig. 9. Impulse response of the learning model, i.e., $\mathcal{F}_{N}^{-1}\left\{\alpha(k) \hat{G}^{-1}(k)\right\}$ $(-)$, shows significant noncausal behavior indicating NMP dynamics.

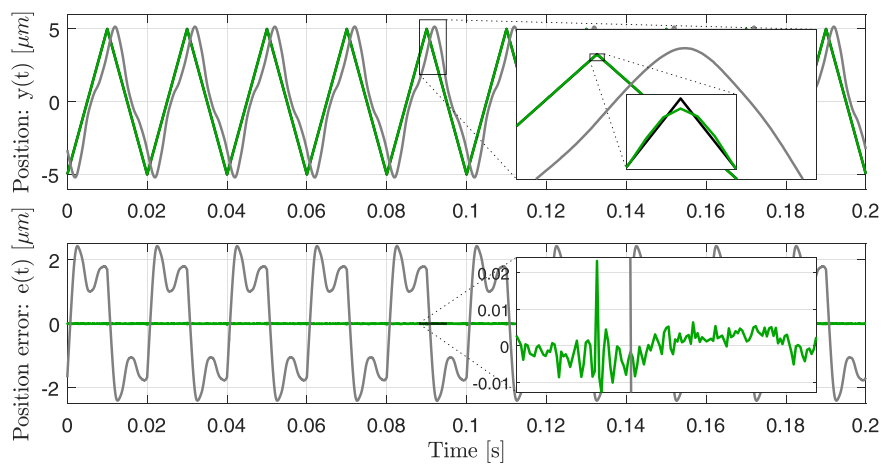

Fig. 10. (Top) Reference ( - ), the initial output $y_{0}(t)(-)$, and the output after 30 FT-IIC iterations $y_{30}(t)(-)$. (Bottom) Similar plot of the tracking error, which shows that converged error is about a factor 100 smaller.

This requires subsequent exploratory experiments to empirically check for convergence and is, therefore, not further pursued here. To get a feel for the time-domain properties of the inverse model, its periodic impulse response, $\mathcal{F}_{N}^{-1}\{\alpha(k) \hat{G}(k)\}$, is displayed in Fig. 9. The response shows a significant noncausal contribution, which in light of Remark 2 implies that the system is NMP.

Performing the next experiment with $Q(k)=1$ and $\alpha(k)$, as shown in Fig. 8, results in a significant performance improvement, as shown in Fig. 10. These plots show the output and tracking error during iterations $i=0$ and $i=30$. This reveals that the peak error is reduced to less than $0.5 \%$ of the reference. In Fig. 11, the root-mean-square (rms) error is shown as a function of the iterations. This shows that after seven iterations the rms has decreased nearly 3 orders in magnitude. During these first iterations, $\left\|e_{i}\right\|_{2}$ can be described by the exponential $0.4^{i}\left\|e_{0}\right\|_{2}$, which shows that the convergence rate of $\left\|e_{i}\right\|_{2}$ is approximately $\kappa^{-1} \approx 1-\alpha_{c}=0.4$. Furthermore, the rms error converges to a level which is very close to the mean rms of the nonrepeating components of the error, which is estimated by averaging 30 identical experiments. In Fig. 12, the PSDs of $e_{0}$ and $e_{30}$ are shown. The PSD of $e_{0}(t)$ reveals contributions at $50+100 \cdot n \mathrm{~Hz}$ and at $100 \cdot n \mathrm{~Hz}$, where the latter are induced

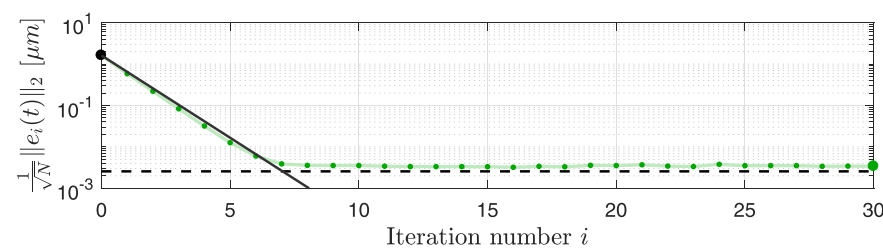

Fig. 11. rms tracking error as a function of the iterations $(\bullet)$ shows significant performance improvement. For the first seven iterations, its behavior is proportional to the exponential $0.4^{i}(-)$, and the asymptotic value is lower bounded by the mean rms of the nonrepeating components of the error $(---)$.

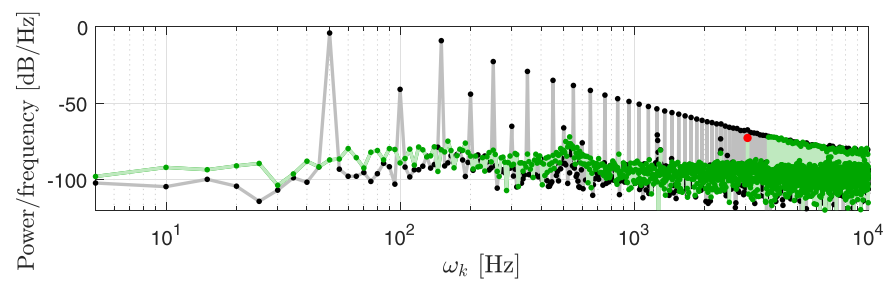

Fig. 12. PSD of $e_{0}(t)(\bullet)$ and $e_{30}(t)(\bullet)$. The point $(\bullet)$ indicates the contribution at $\omega_{k}^{*}=3050 \mathrm{~Hz}$.

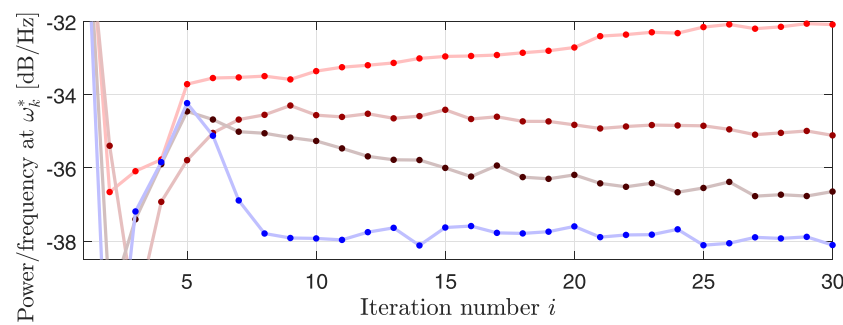

Fig. 13. PSD of the tracking error at $\omega_{k}^{*}=3050 \mathrm{~Hz}$ during the tuning procedure, where $Q=1$ and $\alpha=0.05(\bullet), \alpha=-0.01(\bullet)$, and $\alpha=-0.05$ $(\bullet)$ and where $Q=0.5$ and $\alpha=-1(\bullet)$. This shows that by taking $Q\left(\omega_{k}^{*}\right)<1$, the convergence speed can be increased, even when the model $\hat{G}$ is artificially made to be inaccurate by choosing $\alpha\left(\omega_{k}^{*}\right)$ large. Each line represents the average of 40 experiments.

by nonlinearities, since these do not appear in $R(k)$. The PSD of $e_{30}(t)$ confirms that disturbance rejection is achieved for all frequencies at which $\alpha(k) \neq 0$. Notably, the largest component occurs at $\omega_{k}^{*}=3050 \mathrm{~Hz}$, since $\alpha\left(k^{*}\right)=0$.

This frequency is specifically treated by executing steps 1 and 2 of Design Guideline 1. The result is shown in Fig. 13, where each line represents the average of 40 experiments.

1) By taking $\alpha\left(k^{*}\right)=0.05$, the PSD of the error diverges.

2) To achieve convergence, $\alpha\left(k^{*}\right)$ is reduced, and the sign of $\alpha\left(k^{*}\right) \hat{G}\left(k^{*}\right)$ is changed by taking $\alpha\left(k^{*}\right)=-0.01$. This results in slow convergence.

3) The convergence speed in increased by increasing $\alpha\left(k^{*}\right)$. It turns out that $\alpha\left(k^{*}\right)=-0.05$ yields the best result for $Q=1$. The convergence is relatively slow.

Since $\alpha\left(k^{*}\right)$ is relatively small, step 3 can be performed to increase the convergence speed by taking $Q\left(k^{*}\right)<1$. Moreover, since convergence is only achievable for $-0.05<\alpha<0$, a large perturbation $\Delta\left(k^{*}\right)$ can be simulated by taking $\alpha\left(k^{*}\right)=-1$. By taking $Q\left(k^{*}\right)=0.5$, convergence is nevertheless achieved 

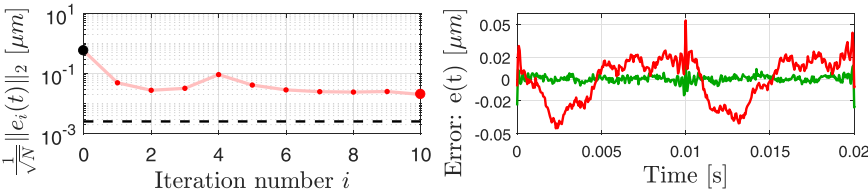

Fig. 14. (Top left) rms error of data-driven FT-IIC $(\bullet)$ shows performance improvement, and irregular behavior at $i=4$ (-). (Top right) Data-driven FT-IIC error is larger than the FRF-based FT-IIC error $((-))$ at $i=10$.

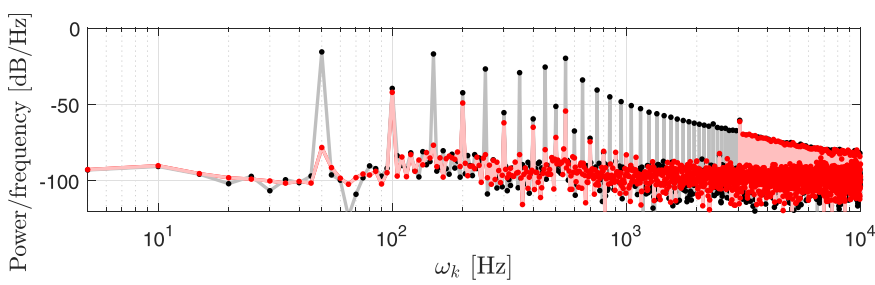

Fig. 15. PSD of $e_{0}(t)(\bullet)$ and $e_{30}(t)(\bullet)$ for data-driven FI-IIC.

and at a higher rate, as is clear from Fig. 13. This shows that by incorporating the robustness coefficient $Q$, superior performance is achieved with respect to pre-existing IIC [19].

\section{Comparison to Data-Driven Learning}

In this section, the FRF-based approach is compared to a datadriven approach. The latter is obtained by setting $Q(k)=1$ and replacing $\hat{G}$ by $\hat{G}_{i}=\left(U_{i}(k)+C_{f b}\left(e^{j \omega_{k}} R(k)\right)\right) Y_{i}(k)^{-1}$, where $U_{i}$ and $Y_{i}$ result from iteration $i$ [22]. To avoid learning transients for small $\left|Y_{i}\right|$, an exponentially decreasing learning gain is used, i.e., $\alpha(k)=e^{-i / i_{\max }} \forall k$. The resulting performance is shown in Fig. 14. Clearly, the achieved performance is inferior, which is mainly due to the fact that the data-driven approach cannot reject the nonlinear disturbances that appear at those frequencies where $R(k)=0$, as is apparent by comparing Figs. 12 and 15 . Furthermore, the convergence behavior, as shown in Fig. 14, displays a learning transient at $i=4$ despite the exponentially decreasing $\alpha(k)$. This indicates that more rigorous measures are required to ensure transient-free convergence, as is a subject of ongoing research [33].

In summary, the FT-IIC method is applied to a nanopositioner, which shows that broadband disturbance rejection can be achieved by using nonparametric FRF models. By following the proposed design guidelines, rapid convergence and robustness against plant uncertainties is achieved.

\section{CONCLUSION}

In this paper, the FT-IIC method is developed, which iteratively improves the tracking performance of a stable LTI process by using a nonparametric FRF estimate. It is shown that under mild conditions, FRF-based inversion results in bounded inputs, even if the system possesses right-half-plane zeros. In contrast to pre-existing IIC approaches, the developed approach allows for robustness against plant variations. This is facilitated by the derivation of design guidelines based on steady-state performance, monotonic convergence, and robustness. Furthermore, a finite-time analysis of the batch-to-batch and continuous implementations reveals that the effect of transients can readily be mitigated by waiting. The potential of the proposed approach is confirmed in experiments by application to a nanopositioner.

\section{APPENDIX A \\ PROOF OF LEMMA 1}

Proof: Since $R(\omega)$ exists and since $G^{-1}\left(e^{j \omega}\right) R(\omega)$ is bounded on $(-\pi, \pi], U(\omega)=\left[G\left(e^{j \omega}\right)\right]^{-1} \mathcal{F}_{\infty}\{r\}$ is integrable on $(-\pi, \pi]$, and hence, (4) exists and $u \in l_{\infty}$. Furthermore, since $\mathcal{F}_{\infty}\{x(k+1)\}=e^{j \omega} \mathcal{F}_{\infty}\{x(k)\}$ [28, Sec. 2.2], the DTFT of (1a) equals $e^{j \omega} X(\omega)=A X(\omega)+B U(\omega)$, and since $G$ has no poles on the unit disc, $X(\omega)=$ $\left(e^{j \omega} I-A\right)^{-1} B U(\omega)$ is unique and bounded for any $U(\omega)$. Next, consider the DTFT of (1b) $Y(\omega)=C X(\omega)+$ $D U(\omega)=\left(C\left[e^{j \omega} I-A\right]^{-1} B+D\right) U(\omega)$, and hence, $Y(\omega)=$ $G\left(e^{j \omega}\right) U(\omega)=G\left(e^{j \omega}\right)\left[G\left(e^{j \omega}\right)\right]^{-1} R(\omega)$, which shows that (1) holds, and $y(k)=r(k) \forall k \in \mathbb{Z}$.

\section{APPENDIX B \\ PROOF OF LEMMA 2}

Proof: Combining (6) with $E_{i}=R-Y_{i}$ and using $\hat{G}(k)$ to model $G\left(e^{j \omega_{k}}\right)$ leads to $\hat{E}_{i+1}=\hat{E}_{i}-\hat{G}\left(U_{i+1}-U_{i}\right)$. Substituting this into the optimality condition $\frac{\partial \mathcal{J}}{\partial U_{i+1}}=0$ results in (3) with $Q(k)$ and $\alpha(k)$ given by (7).

\section{APPENDIX C \\ PROOF OF LEMMA 3}

Proof: The true error is given by $E_{i+1}=R_{v}-G U_{i+1}$. Substituting the optimal FT-IIC update law (3) into this relation gives $E_{i+1}=R_{v}-Q G\left(U_{i}+\alpha \hat{G}^{-1} E_{i}\right)=R_{v}-$ $Q G U_{i}-Q \alpha G \hat{G}^{-1} E_{i}$. Rewriting $E_{i}$ gives $G U_{i}=R_{v}-E_{i}$. Substitution of the latter in $E_{i+1}$ leads to $E_{i+1}=(1-Q) R_{v}+$ $Q\left(1-\alpha G \hat{G}^{-1}\right) E_{i}$, which at the fixed point $E_{i+1}=E_{i} \triangleq E_{\infty}$ results in (8).

\section{APPENDIX D \\ ProOF OF LEMMA 4}

Proof: Define $\gamma \triangleq\left(1-\alpha \hat{G}^{-1} G\right)$ and substitute (8) and $E_{i+1}$, as in the proof of Lemma 3 , in $\left|E_{i+1}-E_{\infty}\right|$ to yield

$$
\begin{aligned}
\left|E_{i+1}-E_{\infty}\right| & =\left|(1-Q) R_{v}+Q \gamma E_{i}-\frac{(1-Q)}{1-Q \gamma} R_{v}\right| \\
& =\left|Q \gamma\left(E_{i}-\frac{(1-Q)}{1-Q \gamma} R_{v}\right)\right|=\kappa\left|E_{i}-E_{\infty}\right| .
\end{aligned}
$$

Recalling Definition 1 completes the proof.

\section{APPENDIX E \\ PROOF OF LEMMA 5}

Proof: For $\tau<1, \varphi \in \mathbb{C}_{>0}$ and $\mathfrak{R}\{\varphi\}<2$, which implies that (11) is satisfied for all $\alpha \in(0,1]$. For $\tau \geq 1, \varphi \in \mathbb{C}$, i.e., the phase of $\varphi$ is not restricted and consequently $\nexists \alpha$ such that 
(11) is satisfied. For $\alpha=1,(11)$ results in

$$
\kappa(k)=\left|Q(k) \hat{G}^{-1}(k) \Delta(k)\right| \leq\left|Q(k) \frac{1}{\tau(k)}\right| .
$$

Hence, (11) is satisfied $\forall \Delta \in \Delta$ if (12) holds.

\section{APPENDIX F \\ PROOF OF LEMMA 6}

Proof: Restrict the input to be periodic, i.e., $\bar{u}_{i}=\bar{u}$, and assume that the system is in the steady state, i.e., $x(N(n+$ $1))=x(N n)$. Substituting this identity into (13a) combined with (13b) and $x\left(t_{0}\right)=x_{0}$ yields $\bar{e}_{i}=\bar{r}-J_{p} \bar{u}_{i}$, where the periodic response matrix $J_{p}$ is given by (15). Employing the matrix push-through identity shows that $J_{p}$ is circulant. Since $\mathbb{G}_{m}$ is a diagonal matrix, $\mathcal{T} \triangleq \mathcal{F}^{H} \mathbb{G}_{m} \mathcal{F}$ is also a circulant matrix by property of $\mathcal{F}$ [35, Sec. 4.8.2]. Hence, it suffices to compare a single row of $J_{p}$ and $\mathcal{T}$. Using $\mu^{(N-k) i}=$ $\bar{\mu}^{k i}$ and $\frac{1}{N} \sum_{k=0}^{N-1} e^{j \omega_{k} h}\left(e^{j \omega_{k}}-A\right)^{-1}=\left(I-A^{N}\right)^{-1} A^{N-h-1}$, which is validated by direct computation, shows that $J_{p}=\mathcal{T}=$ $\mathcal{F}^{H} \mathbb{G}_{m} \mathcal{F}$.

\section{APPENDIX G \\ PROOF OF THEOREM 2}

Batch-to-batch: Consider $\bar{e}_{i}$, which results from the output after waiting for $w$ periods, as is given by (17), i.e.,

$$
\bar{e}_{i}=\bar{r}-\bar{y}_{i}^{\langle w\rangle}=\bar{r}-H F^{w} x(0)-J_{t} \bar{u}_{i} .
$$

Substitution into (16) yields

$$
\begin{aligned}
\bar{u}_{i+1} & =\mathcal{Q} \bar{u}_{i}+\mathcal{Q} \Lambda \hat{J}_{p}^{-1}\left(\bar{r}-H F^{w} x(0)-J_{t} \bar{u}_{i}\right) \\
& =Z \bar{u}_{i}+\mathcal{Q} \Lambda \hat{J}_{p}^{-1}\left(\bar{r}-H F^{w} x(0)\right) .
\end{aligned}
$$

This first-order linear recursion is stable iff $\bar{\rho}(Z)<1$. In addition, setting $\bar{u}_{i+1}=\bar{u}_{i}=\bar{u}_{\infty}$ in (21) results in the fixed point $u_{\infty}=(I-Z)^{-1} \Lambda \hat{J}_{p}^{-1}\left(\bar{r}-H F^{w} x(0)\right)$, and it follows readily that $\left\|\bar{u}_{i+1}-u_{\infty}\right\|_{2}=\|Z\|_{2}\left\|\bar{u}_{i}-u_{\infty}\right\|_{2}$. Recalling Definition 1, $\bar{u}_{i}$ converges monotonically in $\|.\|_{2}$ if $\|Z\|_{2}=\bar{\sigma}(Z)<1$. In case $\hat{G}(k)=G\left(e^{j \omega_{k}}\right)$, and if $\alpha(k)=1 \forall k$, then it holds that $\Lambda=I$ and $\hat{J}_{p}=J_{p}$. Consequently, $Z=\mathcal{Q}\left(I-J_{p}^{-1} J_{t}\right)$. Consider $J_{t}$, given by (18), $J_{t}=H \sum_{j=0}^{w-1} F^{j} M+J$, which can be written as $J_{t}=H \sum_{j=0}^{\infty} F^{j} M+J-H \sum_{j=w}^{\infty} F^{j} M$, which is identical to $J_{t}=H \sum_{j=0}^{\infty} F^{j} M+J-H F^{w} \sum_{j=0}^{\infty} F^{j} M$. Next, use that $\sum_{j=0}^{\infty} F^{j}=(I-F)^{-1}$, which holds if $\bar{\rho}(F)<1$ [35, Sec. 11.5.6], which holds since $F=A^{N}$ where $\bar{\rho}(A)<$ 1. Hence, $J_{t}=J_{p}-H F^{-w}(I-F)^{-1} M$, where $J_{p}$ is given by (14). Consequently, $Z=\mathcal{Q}\left(I-J_{p}^{-1}\left(J_{p}-H F^{-w}(I-\right.\right.$ $\left.F)^{-1} M\right)$ ), which is identical to (20), thereby completing the proof.

Continuous: Consider $\bar{e}_{i}$ that results after waiting for $w$ periods since the previous input update, as is given by (17), i.e., $\bar{e}_{i}=\bar{r}-\bar{y}^{\langle n+w\rangle}=\bar{r}-H F^{w} x(N n)-J_{t} \bar{u}^{\langle n\rangle}$. Next, let $\bar{u}^{\langle w+n\rangle}$ be given by $\bar{u}_{i+1}$ as given by (16), i.e.,

$$
\begin{aligned}
\bar{u}^{\langle w+n\rangle} & =\mathcal{Q} \bar{u}^{\langle n\rangle}+\mathcal{Q} \Lambda \hat{J}_{p}^{-1}\left(\bar{r}-H F^{w} x(N n)-J_{t} \bar{u}^{\langle n\rangle}\right) \\
& =Z \bar{u}^{\langle n\rangle}+\mathcal{Q} \Lambda \hat{J}_{p}^{-1}\left(\bar{r}-H F^{w} x(N n)\right) .
\end{aligned}
$$

In addition, $x(N(n+w))$ follows by recursion of (13a)

$$
x(N(n+w))=F^{w} x(N n)+\sum_{j=0}^{w-1} F^{j} M \bar{u}^{\langle n\rangle} .
$$

Hence, a realization of the lifted closed-loop dynamics is

$$
\xi(n+w)=\mathcal{A} \xi(n)+\left[\begin{array}{c}
0 \\
\mathcal{Q} \Lambda \hat{J}_{p}^{-1}
\end{array}\right] \bar{r}, \quad \xi(n)=\left[\begin{array}{c}
x(N n) \\
\bar{u}^{\langle n\rangle}
\end{array}\right]
$$

where $\mathcal{A}$ is given by (19). The closed dynamics are stable iff $\bar{\rho}(\mathcal{A})<1$, which concludes the proof.

\section{REFERENCES}

[1] Y. Shan and K. K. Leang, "Design and control for high-speed nanopositioning: Serial-kinematic nanopositioners and repetitive control for nanofabrication," IEEE Control Syst. Mag., vol. 33, no. 6, pp. 86-105, Dec. 2013.

[2] J. Bolder, T. Oomen, S. Koekebakker, and M. Steinbuch, "Using iterative learning control with basis functions to compensate medium deformation in a wide-format inkjet printer," Mechatronics, vol. 24, pp. 944-953, 2014.

[3] D. Hoelzle, A. Alleyne, and A. W. Johnson, "Basis task approach to iterative learning control with applications to micro-robotic deposition," IEEE Trans. Control Syst. Technol., vol. 19, no. 5, pp. 1138-1148, Sep. 2011.

[4] S. Hara, Y. Yamamoto, T. Omata, and M. Nakano, "Repetitive control system: A new type servo system for periodic exogenous signals," IEEE Trans. Autom. Control, vol. 33, no. 7, pp. 659-668, Jul. 1988.

[5] Y. R. Teo, A. J. Fleming, A. A. Eielsen, and J. T. Gravdahl, "A simplified method for discrete-time repetitive control using model-less finite impulse response filter inversion,” J. Dyn. Syst., Meas., Control, vol. 138, no. 8, 2016, Art. no. 081002.

[6] D. Bristow, M. Tharayil, and A. Alleyne, "A survey of iterative learning control,” IEEE Control Syst. Mag., vol. 26, no. 3, pp. 96-114, Jun. 2006.

[7] S. Mishra, K. L. Barton, A. G. Alleyne, P. M. Ferreira, and J. A. Rogers, "High-speed and drop-on-demand printing with a pulsed electrohydrodynamic jet," J. Micromech. Microeng., vol. 20, no. 9, 2010, Art. no. 095026.

[8] F. Boeren, A. Bareja, T. Kok, and T. Oomen, "Frequency-domain ILC approach for repeating and varying tasks: With application to semiconductor bonding equipment," IEEE/ASME Trans. Mechatronics, vol. 21, no. 6, pp. 2716-2727, Dec. 2016.

[9] L. Blanken, F. Boeren, D. Bruijnen, and T. Oomen, "Batch-to-batch rational feedforward control: From iterative learning to identification approaches, with application to a wafer stage," IEEE/ASME Trans. Mechatronics, vol. 22, no. 2, pp. 826-837, Apr. 2017.

[10] K. Moore, Iterative Learning Control for Deterministic Systems. London, U.K.: Springer-Verlag, 1993.

[11] L. Blanken, T. Hazelaar, S. Koekebakker, and T. Oomen, "Multivariable repetitive control design framework applied to flatbed printing with continuous media flow," in Proc. IEEE 56th Annu. Conf. Decis. Control, 2017, pp. 4727-4732.

[12] J. van Zundert and T. Oomen, "On inversion-based approaches for feedforward and ILC," Mechatronics, vol. 50, pp. 282-291, 2018.

[13] S. Devasia, D. Chen, and B. Paden, "Nonlinear inversion-based output tracking," IEEE Trans. Autom. Control, vol. 41, no. 7, pp. 930-942, Jul. 1996.

[14] G. Zeng and L. Hunt, "Stable inversion for nonlinear discrete-time systems," IEEE Trans. Autom. Control, vol. 45, no. 6, pp. 1216-1220, Jun. 2000.

[15] M. Tomizuka et al., "Zero phase error tracking algorithm for digital control,” J. Dyn. Syst. Meas., Control, vol. 109, no. 1, pp. 65-68, 1987.

[16] M. Tomizuka and W. Messner, "Cancellation of discrete time unstable zeros by feedforward control," J. Dyn. Syst., Meas., Control, vol. 116, pp. 33-38, 1994.

[17] Q. Zou, "Optimal preview-based stable-inversion for output tracking of nonminimum-phase linear systems," Automatica, vol. 45, no. 1, pp. 230-237, 2009.

[18] R. de Rozario, A. J. Fleming, and T. Oomen, "Iterative control for periodic tasks with robustness considerations, applied to a nanopositioning stage," IFAC-PapersOnLine, vol. 49, no. 21, pp. 623-628, 2016. 
[19] S. Tien, Q. Zou, and S. Devasia, "Iterative control of dynamics-couplingcaused errors in piezoscanners during high-speed AFM operation," IEEE Trans. Control Syst. Technol., vol. 13, no. 6, pp. 921-931, Nov. 2005.

[20] M. Norrlöf and S. Gunnarsson, "Time and frequency domain convergence properties in iterative learning control," Int. J. Control, vol. 75, no. 14, pp. 1114-1126, 2002.

[21] Y. Wu and Q. Zou, "Iterative control approach to compensate for both the hysteresis and the dynamics effects of piezo actuators," IEEE Trans. Control Syst. Technol., vol. 15, no. 5, pp. 936-944, Sep. 2007.

[22] K.-S. Kim and Q. Zou, "A modeling-free inversion-based iterative feedforward control for precision output tracking of linear time-invariant systems," IEEE/ASME Trans. Mechatronics, vol. 18, no. 6, pp. 1767-1777, Dec. 2013.

[23] Y. Yan, H. Wang, and Q. Zou, "A decoupled inversion-based iterative control approach to multi-axis precision positioning: $3 \mathrm{~d}$ nanopositioning example," Automatica, vol. 48, no. 1, pp. 167-176, 2012.

[24] Y. Wu, Q. Zou, and C. Su, "A current cycle feedback iterative learning control approach for AFM imaging," IEEE Trans. Nanotechnol., vol. 8, no. 4, pp. 515-527, Jul. 2009

[25] G. M. Clayton, S. Tien, K. K. Leang, Q. Zou, and S. Devasia, "A review of feedforward control approaches in nanopositioning for high-speed SPM," J. Dyn. Syst., Meas., Control, vol. 131, no. 6, 2009, Art. no. 061101.

[26] J. Bechhoefer, "Feedforward control of a piezoelectric flexure stage for AFM,” in Proc. Amer. Control Conf., Jun. 2008, pp. 2703-2709.

[27] T. Sogo, "On the equivalence between stable inversion for nonminimum phase systems and reciprocal transfer functions defined by the two-sided laplace transform," Automatica, vol. 46, no. 1, pp. 122-126, 2010.

28] L. R. Rabiner and B. Gold, Theory and Application of Digital Signal Processing. Englewood Cliffs, NJ, USA: Prentice-Hall, 1975.

[29] A. Bazaei, Y. K. Yong, and S. R. Moheimani, "Combining spiral scanning and internal model control for sequential AFM imaging at video rate," IEEE/ASME Trans. Mechatronics, vol. 22, no. 1, pp. 371-380, Feb. 2017.

[30] A. J. Fleming and K. K. Leang, Design, Modeling and Control of Nanopositioning Systems. New York, NY, USA: Springer, 2014.

[31] R. Pintelon and J. Schoukens, System Identification: A Frequency Domain Approach. Hoboken, NJ, USA: Wiley, 2012.

[32] R. de Rozario, T. Oomen, and M. Steinbuch, "Iterative learning control and feedforward for LPV systems: Applied to a position-dependent motion system," in Proc. Amer. Control Conf., 2017, pp. 3518-3523.

[33] R. de Rozario and T. Oomen, "Improving transient learning behavior in model-free inversion-based iterative control with application to a desktop printer," in Proc. 15th Int. Workshop Adv. Motion Control, 2018 pp. $455-460$.

[34] G. Pipeleers and K. L. Moore, "Unified analysis of iterative learning and repetitive controllers in trial domain," IEEE Trans. Autom. Control, vol. 59, no. 4, pp. 953-965, Apr. 2014.

[35] G. H. Golub and C. F. Van Loan, Matrix Computations, vol. 3. Baltimore, MD, USA: Johns Hopkins Univ. Press, 2013.

[36] D. Roover, O. H. Bosgra, and M. Steinbuch, "Internal-model-based design of repetitive and iterative learning controllers for linear multivariable systems," Int. J. Control, vol. 73, no. 10, pp. 914-929, 2000

[37] J. Liu, B. Yan, and Q. Zou, "Optimal time-distributed fast Fourier transform: Application to online iterative learning control-experimental highspeed nanopositioning example," Mechatronics, vol. 41, pp. 114-124, 2017.

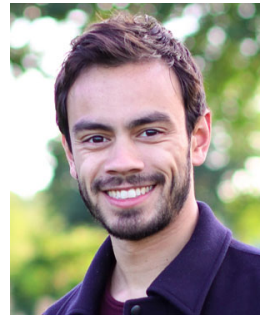

Robin de Rozario received the M.Sc. degree (cum laude) in mechanical engineering in 2015 from the Eindhoven University of Technology, Eindhoven, The Netherlands, where he is currently working toward the Ph.D. degree with the Control Systems Technology Group, Department of Mechanical Engineering.

His research interests include identification and learning control for high-performance motion systems.

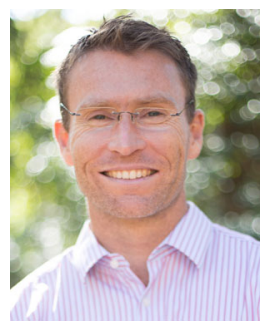

Andrew Fleming received the Bachelor of Electrical Engineering and Ph.D. degrees from The University of Newcastle, Callaghan NSW, Australia, in 2000 and 2004, respectively.

$\mathrm{He}$ is currently an Australian Research Council Future Fellow and Director of the Precision Mechatronics Lab, The University of Newcastle. $\mathrm{He}$ has coauthored three books and more than 100 journal and conference papers. He holds several patent applications. His research interests include nanofabrication, microrobotics, meteorological sensing, nanopositioning, and high-speed scanning probe microscopy.

Dr. Fleming is the recipient of the IEEE TRANSACTIONS ON CONTROL SyStems TECHNOLOGY Outstanding Paper Award, The University of Newcastle Researcher of the Year Award, and the Faculty of Engineering and Built Environment Award for Research Excellence. He received the Newcastle Innovation Rising Star Award for Excellence in Industrial Engagement in 2012.

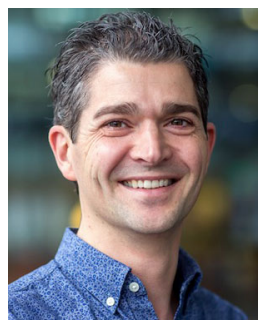

Tom Oomen received the M.Sc. (cum laude) and Ph.D. degrees from the Eindhoven University of Technology, Eindhoven, The Netherlands, 2005 and 2010, respectively.

He held visiting positions with the KTH Royal Institute of Technology, Stockholm, Sweden, and with The University of Newcastle, Callaghan, NSW, Australia. He is currently an Associate Professor with the Department of Mechanical Engineering, Eindhoven University of Technology. His research interests include data-driven modeling, learning, and control, with applications in precision mechatronics.

Dr. Oomen is a recipient of the Corus Young Talent Graduation Award, the 2015 IEEE TRANSACTIONS ON CONTROL SYSTEMS TECHNOLOGY Outstanding Paper Award, and the 2017 IFAC Mechatronics Best Paper Award. He is recipient of a Veni and Vidi Personal Grant. $\mathrm{He}$ is an Associate Editor for the IEEE CONTROL SYSTEMS LETTERS, IFAC Mechatronics, and the IEEE TRANSACTIONS ON CONTROL SYSTEMS TECHNOLOGY. He is a member of the Eindhoven Young Academy of Engineering. 